\title{
Coma revealed as an extended hard X-rays source by INTEGRAL IBIS/ISGRI
}

\author{
M. Renaud ${ }^{1,2}$, G. Bélanger ${ }^{1,2}$, J. Paul ${ }^{1,2}$, F. Lebrun ${ }^{1,2}$, and R. Terrier ${ }^{2}$ \\ 1 Service d'Astrophysique, CEA-Saclay, 91191 Gif-Sur-Yvette, France \\ e-mail: mrenaud@cea.fr \\ 2 APC-UMR 7164, 11 place M. Berthelot, 75231 Paris, France
}

Received 7 March 2006 / Accepted 26 April 2006

ABSTRACT

\begin{abstract}
Aims. We report the INTEGRAL/IBIS observations of the Coma Cluster in the hard X-ray/soft $\gamma$-ray domain. Methods. Since the Coma Cluster appears as an extended source, its global intensity and significance cannot be directly extracted with standard coded mask analysis. We used the method of imaging the extended sources with a coded mask telescope developed by Renaud et al. (2006).

Results. The imaging capabilities and the sensitivity of the IBIS/ISGRI coded mask instrument allows us to identify for the first time the site of the emission above $\sim 15 \mathrm{keV}$. We have studied the Coma Cluster morphology in the $18-30 \mathrm{keV}$ band and found that it follows the prediction based on X-ray observations. We also bring constraints on the non-thermal mechanism contribution at higher energies.
\end{abstract}

Key words. galaxies: clusters: Coma Cluster - methods: data analysis - gamma rays: observations - X-rays: galaxies: clusters

\section{Introduction}

Clusters of galaxies are the largest bound structures in the visible universe, and amongst the most luminous ones. This makes them important cosmological probes. The intercluster medium (ICM) is heated to high temperatures by the initial collapse. In addition, it is believed that as groups and smaller clusters of galaxies merge to form larger ones, violent shocks compress and heat the intercluster medium to X-ray emitting temperatures $\left(10^{7}-10^{8} \mathrm{~K}\right)$. These shocks could also drive the acceleration of ions and electrons to relativistic energies, thereby producing a spectrum extending from the radio to the $\gamma$-ray domains through processes such as synchrotron emission and inverse Compton (hereafter, IC) scattering.

The observations of diffuse radio halos from cluster cores (Giovannini et al. 1993), and the more recent reported detection of extreme ultraviolet (EUV) and hard X-ray (HXR) emission from clusters (Bowyer et al. 1999; Fusco-Femiano et al. 1999; Rephaeli et al. 1999) seem to point to the possible presence of non-thermal and/or supra-thermal particles. Although the origin of the radio halos is most probably synchrotron emission from high energy electrons, that of the EUV photons and hard Xrays has been the source of much debate and is still uncertain. Sarazin (1999) suggested that the extreme UV and hard X-rays could arise from IC scattering of the energetic, synchrotron producing electrons off the cosmic microwave background radiation (see also Bykov et al. 2000 for a detailed discussion of nonthermal high energy emission in galaxy clusters). Alternatively, this emission could also be due to bremsstrahlung radiation of suprathermal electrons accelerated by turbulent gas dynamics in the ICM (Ensslin et al. 1999; Sarazin \& Kempner 2000). If the HXR cluster component is confirmed, then the question remains: is it thermal or non-thermal in origin?
Thus far, X-ray imaging observations of clusters such as Coma, have shown that the emission in the X-ray range is predominantly thermal, originating from bremsstrahlung in the hot ICM. BeppoSAX and RXTE detected a hard X-ray excess that extends beyond $10 \mathrm{keV}$ and apparently deviates from the steep thermal spectrum expected from pure bremsstrahlung emission (Fusco-Femiano et al. 1999, 2004; Rephaeli et al. 1999; Rephaeli $\&$ Gruber 2002). Given that these observations were carried out with non-imaging instruments, contamination from point sources contained in their field of view is not unlikely. Moreover, a recent second look at the BeppoSAX data on Coma seems to show that there is no evidence of a HXR excess (Rossetti \& Molendi 2004). This debate can only be convincingly resolved and put to rest through sensitive imaging observations at high energies beyond the thermally dominated X-ray emission.

In this letter, we report on hard X-ray/soft $\gamma$-ray observations of the Coma Cluster with the IBIS/ISGRI (Ubertini et al. 2003; Lebrun et al. 2003) coded mask instrument onboard INTEGRAL (Winkler et al. 2003). A large field of view $\left(29^{\circ} \times 29^{\circ}, 8^{\circ} \times 8^{\circ}\right.$ fully coded), fine imaging capabilities (PSF of $12^{\prime} F W H M$, Gros et al. 2003), and an unprecedented $3 \sigma$ broadband sensitivity of $\sim 1$ mcrab at $20 \mathrm{keV}\left(10^{5} \mathrm{~s}, \Delta E=E / 2\right)$, make IBIS/ISGRI ideal for probing the nature of the HXR excess in the Coma Cluster: the first extended source detected with this instrument.

\section{Observations and data reduction}

The Coma Cluster was observed with INTEGRAL in 2003 during revolutions 36, 71 and 72 . Almost 250 pointings aiming within $13^{\circ}$ of Coma amount to $\sim 500 \mathrm{ks}$. Using these data, Krivonos et al. (2005) demonstrated the ability of IBIS/ISGRI to detect faint extra-galactic sources, and established a catalog of 12 serendipitous sources $(\geq 4 \sigma)$ in addition to the Coma Cluster. 


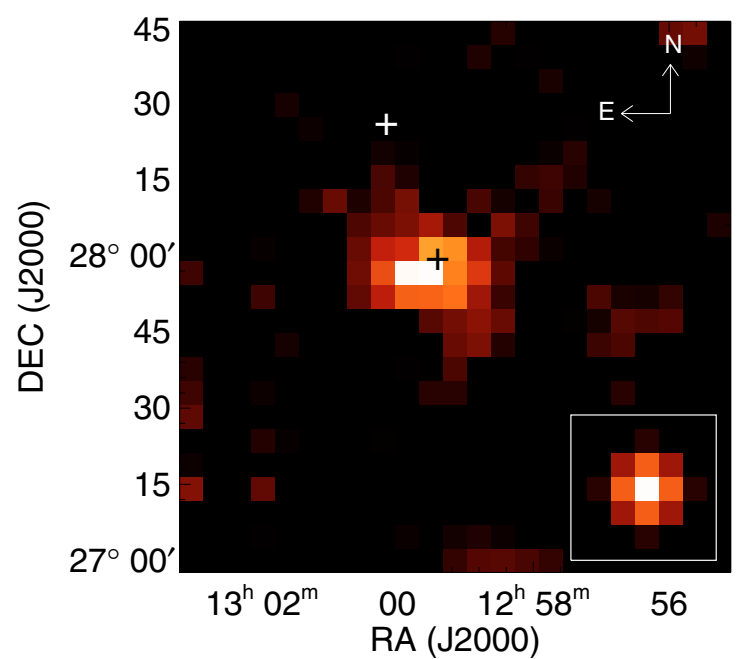

Fig. 1. IBIS/ISGRI significance image of the Coma cluster in the $18-30 \mathrm{keV}$ energy range. The black cross indicates the position of NGC 4874, near the center of the X-ray emission from Coma (Briel et al. 2001). The white cross shows the location of X Comae, a bright Seyfert galaxy at $\sim 30^{\prime}$ from the cluster. The colour scale is linear, with black corresponding to $1 \sigma$ and white to $8 \sigma$. The IBIS SPSF is also shown in the lower right corner.

The authors noted that the source corresponding to the latter was extended, and hence, its flux and significance calculated with the standard analysis were likely inaccurate. Renaud et al. (2006) presented a general method to determine the flux and the total detection significance for any extended celestial source observed through a coded mask. We here apply this method to the study of the spatial and spectral features of the Coma Cluster.

Reconstructed sky images with coded mask telescopes are correlation maps between the detector image and a decoding array derived from the mask pattern. The deconvolution algorithm implemented in the INTEGRAL Off-Line Scientific Analysis (OSA, Goldwurm et al. 2003) is optimized for point-sources and the flux of any source is given by the peak of the SPSF at its position in the correlation map. For this reason, the intensity of an extended source cannot be derived directly using the standard processing. This limitation can be overcome by constructing images of flux per solid angle (i.e. per sky pixel), in which the global flux is given by the sum of intensities over the emissive region. The details of this method are presented in Renaud et al. (2006).

We analyzed the Coma Cluster data with OSA v. 5.0 and obtained individual sky images in the 18-30, 30-50, 50-100 and $100-150 \mathrm{keV}$ energy bands, directly from the standard analysis. We evaluated their quality by measuring the noise given by the width of the distribution of significance values, and then constructed images in flux per sky pixel which were combined to make mosaics in the four energy ranges.

\section{Results}

\subsection{Morphology of the emission}

The IBIS/ISGRI significance map of the Coma Cluster in the energy range $18-30 \mathrm{keV}$ in shown in Fig. 1. We have overlaid the instrument's response to a point-like source of comparable intensity in the bottom right corner of the image. This serves as a morphological comparison, and strongly suggests that the Coma Cluster is indeed an extended source for IBIS/ISGRI. The

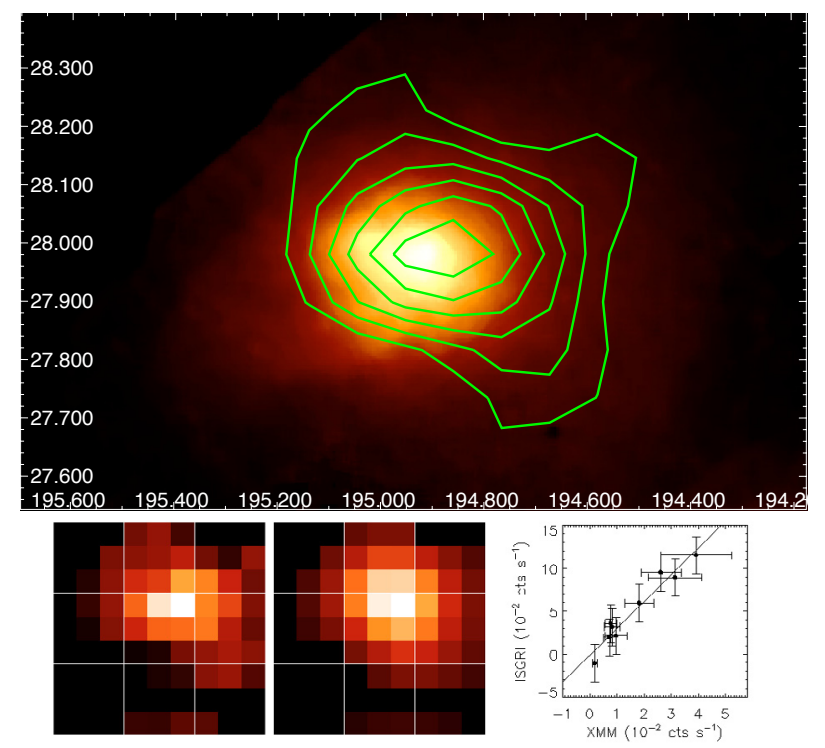

Fig. 2. Top panel: XMM-Newton $0.3-2 \mathrm{keV}$ mosaic image Neumann et al. (2003) with IBIS/ISGRI significance contours in the $18-30 \mathrm{keV}$ energy range, going from $2 \sigma$ to $10 \sigma$. Bottom panel: IBIS/ISGRI image of flux per pixel in the $18-30 \mathrm{keV}$ band centered on the Coma Cluster (left), the predicted morphology based on the XMM-Newton images in intensity and inferred temperature (middle), and the correlation between the $18-30 \mathrm{keV}$ and $0.3-2 \mathrm{keV}$ images (right). The white squares delineate the regions over which the flux was summed for the purpose of correlation study.

maximal pixel value within the extended emission is at the $\sim 8 \sigma$ confidence level, consistent with Krivonos et al. (2005) who noted that it was likely unresolved.

In the X-ray domain, the spectrum of the Coma Cluster is dominated by thermal bremsstrahlung from hot intercluster gas. RXTE observations (Rephaeli \& Gruber 2002) yielded values from which we can derive the expected ratio of non-thermal to thermal emission at higher energies. These are 0.2 and 1.0 in the $18-30 \mathrm{keV}$ and $30-50 \mathrm{keV}$ energy ranges respectively. $X M M-N e w t o n$ observations revealed marked temperature variations (from 4 to $11 \mathrm{keV}$ ) at distances $>10^{\prime}$ from the centre of the cluster (Arnaud et al. 2001; Briel et al. 2001; Neumann et al. 2003). A hot front in the southwest $(k T \sim 11 \mathrm{keV})$ was interpreted as an adiabatic compression due to recent accretion of matter. Within the core, centered on NGC 4874, these authors found a homogeneous temperature distribution of $\sim 8.25 \mathrm{keV}$.

Given that the X-ray emission between 0.3 and $2 \mathrm{keV}$ depends only slightly on the plasma temperatures in the range of those measured in the ICM (Fig. 1 of Arnaud 2005), the XMM-Newton map in this energy range is a good estimate of the ICM gas emissivity. Therefore, we can use the $0.3-2 \mathrm{keV}$ EPIC MOS mosaic image, shown in the top panel of Figure 2 and overlaid with the IBIS/ISGRI $18-30 \mathrm{keV}$ contours, in conjunction with the temperature map of Arnaud et al. (2001) to construct a map of the expected thermal emission in the $18-30 \mathrm{keV}$ band. Moreover, for best accuracy, we computed correction factors, which account for the temperature variations across the cluster, using the mekal model in XSPEC v.11.3. The redshift and abundances were set to $z=0.0231$ and 0.25 , respectively (Arnaud et al. 2001). The relative intensity of each region was weighted with the corresponding correction factor. The final $18-30 \mathrm{keV}$ map, convolved with the IBIS/ISGRI PSF, is shown in the middle bottom panel of Fig. 2. The IBIS/ISGRI 
image shown in Fig. 1 is reproduced in the bottom left panel for reference.

To calculate the correlation coefficient between the IBIS/ISGRI mosaic in the $18-30 \mathrm{keV}$ range and the expected map for the emission in this energy band constructed in the manner described above, the sky pixels over which the flux is summed have to be independent. This is very nearly the case at all scales larger than the size of the SPSF, which is $12^{\prime}$ or 2.5 sky pixels. The white squares in the bottom left and middle panels of Fig. 2 correspond to regions that are 3 pixel (14.'5) by 3 pixels in size, and thus regions that we can consider independent from one another as far as the flux and variance are concerned. By summing the flux per pixel over the nine regions in each map, we obtained two sets of nine intensities. The uncertainties associated with the IBIS/ISGRI map were derived according the prescription of Renaud et al. (2006), and those of the interpolated map were calculated based on an uncertainty of $1 \mathrm{keV}$ on the plasma temperature (Arnaud 2006, priv. comm.).

We found a linear correlation coefficient of $\rho=0.95$, and show the correlation plot in the bottom right panel of Fig. 2 . The probability that a random sample of 9 uncorrelated data points yield a linear correlation coefficient of 0.95 or larger is $8.7 \times 10^{-5}$. This close agreement between the morphologies of the detected and expected thermal emission from the Coma Cluster at energies between 18 and $30 \mathrm{keV}$ brings us to conclude that what is seen in this energy range by IBIS/ISGRI is compatible with an emission of pure thermal origin with no indication of a non-thermal component having a significantly different morphology. A distinction between thermal and nonthermal emission components could be detected only through a fine spectro-imaging analysis on small spatial scales and over the entire emissive region. It is interesting to note that this result adds weight to the temperature variations seen in X-rays by XMM-Newton.

\subsection{Reconstructed global intensity}

We now turn to the question of the global intensity of this emission when the source is considered to be extended. Since instruments such as BeppoSAX/PDS and RXTE are only capable of recording the total emission within their $\sim 1^{\circ} \mathrm{FOV}$, their detection of a HXR tail from the Coma Cluster could not definitively and exclusively be attributed to it. This uncertainty is substantiated by the presence of other sources present in the FOV like $\mathrm{X}$ Comae, a bright Seyfert galaxy discovered by ROSAT/PSPC $\sim 30^{\prime}$ northeast of the Coma Cluster (Dow \& White 1995). Even if X Comae may experience flux variability, Fusco-Femiano et al. (1999) demonstrated that because of its steep spectral index, an unusually strong variability would be necessary in order to have a significant effect on the total detected flux. Hence, its hard X-ray emission would be blended into the global flux and not detected as variable. Nonetheless, since IBIS/ISGRI does not detect an excess above $3 \sigma$ at the position of X Comae in either of the four energy bands defined in Sect. 2, we assume that the spectra obtained by $R X T E$ and BeppoSAX are truly representative of the emission from Coma and that there really exists a HXR tail, despite the ongoing dispute on this issue (Rossetti \& Molendi 2004).

Figure 3 shows the intensity in $18-30 \mathrm{keV}$ range, integrated over circular areas with increasing radii centred on the pixel of maximum intensity located at RA $(\mathrm{J} 2000)=12^{\mathrm{h}} 59^{\mathrm{m}} 31.7^{\mathrm{s}}$ and $\operatorname{Dec}(\mathrm{J} 2000)=27^{\circ} 57^{\prime} 07^{\prime \prime}$ ' 6 . The intensity is shown as the monotonically increasing black curve with error bars. The dashed blue curve represents the total statistical significance ( $\mathrm{S} / \mathrm{N}$ ratio)

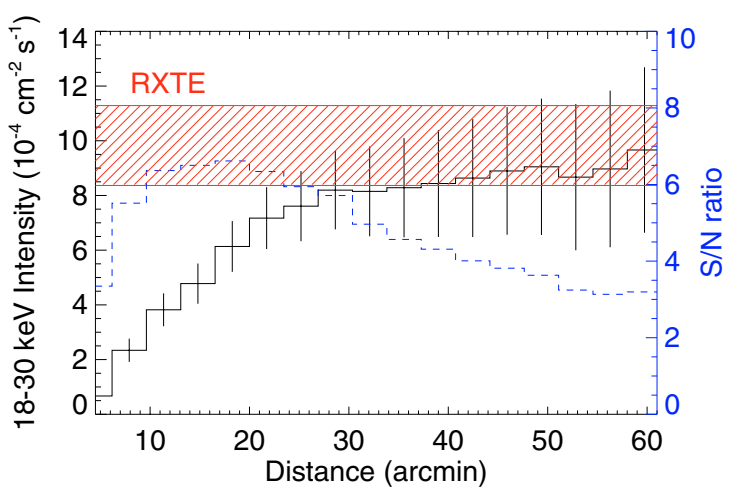

Fig. 3. The 18-30 keV IBIS/ISGRI integrated intensity (left scale, solid line with error bars) and corresponding significance calculated on concentric circles and expressed as a function of radius (right scale, dashed line). The shaded area shows the intensity within the $1 \sigma$ error derived from RXTE observations (Rephaeli \& Gruber 2002; Rossetti \& Molendi 2004).

calculated on the basis of each circular area. This analysis is done without any a priori assumption on the size of the emitting region. The shaded area shows the RXTE flux taken from Rephaeli \& Gruber (2002, Table 1) and Rossetti \& Molendi (2004), whose results are consistent with one another. The best agreement is found for $R=30^{\prime}$. This value is also in line with the extent of the X-ray surface brightness observed by ASCA/GIS (Honda et al. 1996). The IBIS imaging sensitivity was deduced from Crab Nebula observations in each of the four energy bands assuming a Crab spectrum of $9.7 E^{-2.1} \mathrm{~cm}^{-2} \mathrm{~s}^{-1} \mathrm{keV}^{-1}$ (Krivonos et al. 2005).

The treatment in the $30-50 \mathrm{keV}$ energy range was performed in the same way as for the $18-30 \mathrm{keV}$ range, and the flux was summed over the same region but no significant excess was found. Although we expect that at least half of the global intensity in the $30-50 \mathrm{keV}$ band arises from thermal processes, the emission above $50 \mathrm{keV}$ is purely non-thermal. However, no imaging telescopes have observed the Coma at energies $>10 \mathrm{keV}$ and thus the morphology of the yet undetected non-thermal emission above $30 \mathrm{keV}$ is unknown. We derived upper limits for the flux in the $50-100 \mathrm{keV}$ and $100-150 \mathrm{keV}$ ranges in the standard way, relying on the assumption that the source is pointlike $\left(\Phi \lesssim 8^{\prime}\right)$. Even though we have shown that this is not so, these upper limits are a good estimate in the case of a nondetection. The 18-150 keV ISGRI spectrum is shown in Fig. 4. For comparison, we have plotted the RXTE best-fit spectrum of this source (Rephaeli \& Gruber 2002; Rossetti \& Molendi 2004) as the shaded area ( $1 \sigma$ errors), and find that the spectra agree.

\section{Conclusion}

The unequalled imaging capabilities and sensitivity of the IBIS/ISGRI telescope on INTEGRAL give us the opportunity of imaging extended sources in the hard X-ray and soft $\gamma$-ray domains for the first time. We have shown that the morphology of the emission from the Coma Cluster in the $18-30 \mathrm{keV}$ energy range is akin to that in X-rays when the temperature variations measured by XMM-Newton across the cluster's emission are taken into account. There is no evidence for the presence of non-thermal emission arising from a region with the same morphology as that associated with the thermal emission. We find that the integrated intensity is in good agreement with previous RXTE and BeppoSAX observations of Coma. 


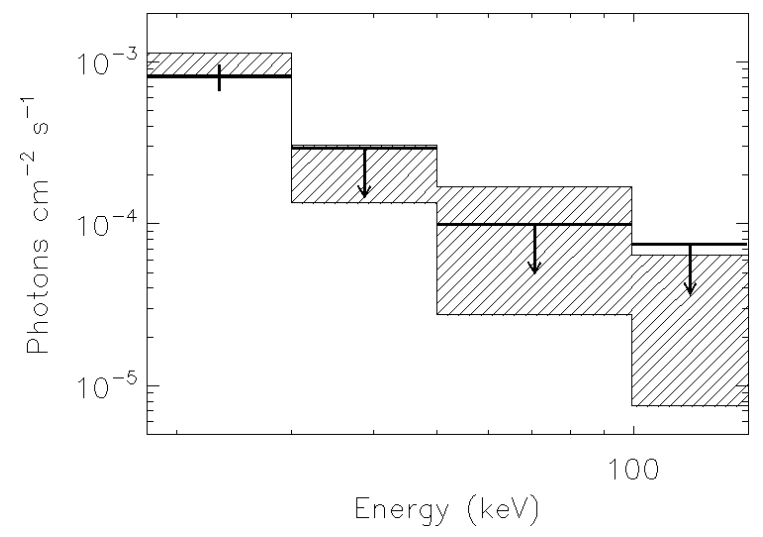

Fig. 4. IBIS/ISGRI spectrum of the Coma Cluster. The upper limit in the $30-50 \mathrm{keV}$ range is calculated assuming that the source has the same shape as it does in the $18-30 \mathrm{keV}$ energy range. At higher energies, the upper limits are derived as for a point-source. The best-fit RXTE spectrum within its $1 \sigma$ uncertainties, is shown as the dashed area. Upper limits are given at the $3 \sigma$ confidence level.

Future INTEGRAL observations will surely reveal more details about the morphology and nature of the emission from Coma. Our analysis was based on the assumption that the morphology of the emission is the same in the $30-50 \mathrm{keV}$ range as it is between 18 and $30 \mathrm{keV}$. Given that the upper limit we quote for the $30-50 \mathrm{keV}$ flux is only a factor of 1.5 above the mean RXTE spectrum, and that non-thermal mechanisms such as IC scattering and non-thermal bremsstrahlung are expected to contribute half the flux in this range, deeper INTEGRAL observations $(\sim 1.5 \mathrm{Ms})$ will permit a fine morphological study of the emissive region. This will provide the opportunity to definitively determine whether there is or not a HXR tail, and if so to identify the site of particle acceleration in the Coma Cluster for the first time.
Acknowledgements. We would like to thank M. Arnaud and D. Neumann for providing the XMM-Newton images and for helpful suggestions. The present work is based on observations with INTEGRAL, an ESA project with instruments and science data center (ISDC) funded by ESA members states (especially the PI countries: Denmark, France, Germany, Italy, Switzerland, Spain, Czech Republic and Poland, and with the participation of Russia and the USA). ISGRI has been realized and is maintained in flight by CEA-Saclay/DAPNIA with the support of the French Space Agency CNES.

\section{References}

Arnaud, M., Aghanim, N., Gastaud, R., et al. 2001, A\&A, 365, L67 Arnaud, M. 2005, summer school lectures at Background Microwave Radiation and Intracluster Cosmology, July 2004, Villa Monastero, Varenna, Italy [arXiv:astro-ph/0508159]

Bowyer, S., Berghöfer, T. W., \& Korpela, E. J. 1999, ApJ, 526, 592 Bykov, A. M., Bloemen, H., \& Uvarov, Yu. A. 2000, A\&A, 362, 886 Briel, U. G., Henry, J. P., Lumb, D. H., et al. 2001, A\&A, 365, L60 Cowan, G. 1998, Statistical Data Analysis (Oxford Science) Dow, K. L., \& White, S. D. M. 1995, ApJ, 439, 113

Enßlin, T. A., Lieu, R., \& Biermann, P. L. 1999, A\&A, 344, 409

Fusco-Femiano, R., Dal Fiume, D., Feretti, L., et al. 1999, ApJ, 513, L21

Fusco-Femiano, R., Orlandini, M., Brunetti, G., et al. 2004, ApJ, 602, L73

Giovannini, G., Feretti, L., Venturi, et al. 1993, ApJ, 406, 399

Goldwurm, A., David, P., Foschini, L., et al. 2003, A\&A, 411, L223

Gros, A., Goldwurm, A., Cadolle-Bel, M., et al. 2003, A\&A, 411, L179

Honda, H., Hirayama, M., Watanabe, M., et al. 1996, ApJ, 473, L71

Krivonos, R., Vikhlinin, A., Churazov, E., et al. 2005, ApJ, 625, 89

Labanti, C., Di Cocco, G., Ferro, G., et al. 2003, A\&A, 411, L149

Lebrun, F., Leray, J.-P., Lavocat, P., et al. 2003, A\&A, 411, L141

Neumann, D. M., Lumb, D. H., Pratt, G. W., \& Briel, U. G. 2003, A\&A, 400, 811

Rephaeli, Y., Gruber, D., \& Blanco, P. 1999, ApJ, 511, L21

Rephaeli, Y., \& Gruber, D. 2002, ApJ, 579, 587

Renaud, M., Lebrun, F., Terrier, R., et al. 2006, A\&A, submitted

Rossetti, M., \& Molendi, S. 2004, A\&A, 414, L41

Sarazin, C. L. 1999, ApJ, 520, 529

Sarazin, C. L., \& Kempner, J. C. 2000, ApJ, 533, 73

Ubertini, P., Lebrun, F., Di Cocco, G., et al. 2003, A\&A, 411, L131

Winkler, C., Courvoisier, T. J.-L., Di Cocco, G., et al. 2003, A\&A, 411, L1 